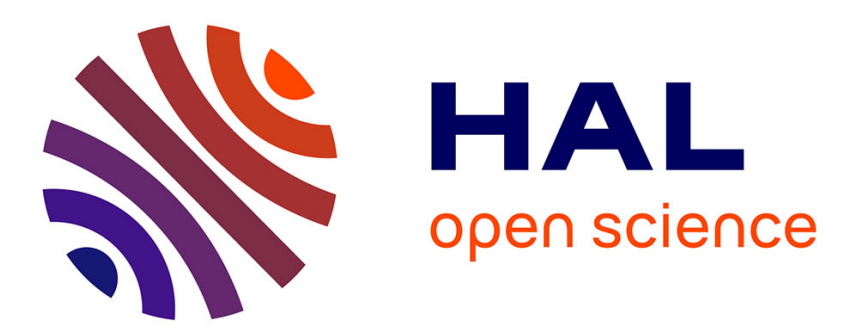

\title{
The heterogeneous nature of slip in ice single crystals deformed under torsion
}

Maurine Montagnat, Jérôme Weiss, Paul Duval, Hélène Brunjail, P Bastie, Javier Gil Sevillano

\section{- To cite this version:}

Maurine Montagnat, Jérôme Weiss, Paul Duval, Hélène Brunjail, P Bastie, et al.. The heterogeneous nature of slip in ice single crystals deformed under torsion. Philosophical Magazine, 2006, 86 (27), pp.4259-4270. 10.1080/14786430500452602 . hal-00513637

\section{HAL Id: hal-00513637 \\ https://hal.science/hal-00513637}

Submitted on 1 Sep 2010

HAL is a multi-disciplinary open access archive for the deposit and dissemination of scientific research documents, whether they are published or not. The documents may come from teaching and research institutions in France or abroad, or from public or private research centers.
L'archive ouverte pluridisciplinaire HAL, est destinée au dépôt et à la diffusion de documents scientifiques de niveau recherche, publiés ou non, émanant des établissements d'enseignement et de recherche français ou étrangers, des laboratoires publics ou privés. 


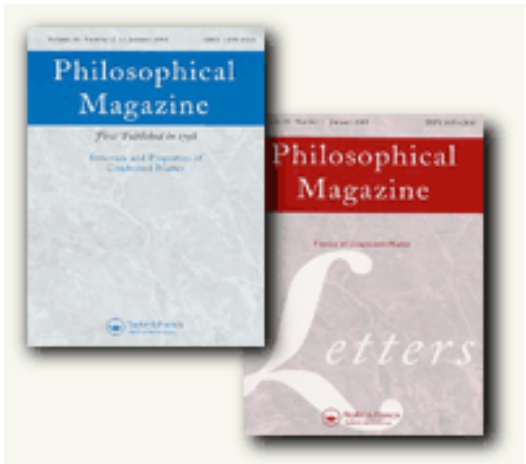

\section{The heterogeneous nature of slip in ice single crystals deformed under torsion}

\begin{tabular}{|c|c|}
\hline Journal: & Philosophical Magazine \& Philosophical Magazine Letters \\
\hline Manuscript ID: & TPHM-05-Aug-0375.R1 \\
\hline Journal Selection: & Philosophical Magazine \\
\hline $\begin{array}{r}\text { Date Submitted by the } \\
\text { Author: }\end{array}$ & 06-Oct-2005 \\
\hline Complete List of Authors: & $\begin{array}{l}\text { Montagnat, Maurine; CNRS, LGGE } \\
\text { Weiss, Jérôme; CNRS, LGGE } \\
\text { Duval, Paul; CNRS, LGGE } \\
\text { Brunjail, Hélène; CNRS, LGGE } \\
\text { Bastie, P; CNRS, Spectrométrie Physique } \\
\text { Gil Sevillano, Javier; Universidad de Navarra, CEIT \& TECNUM }\end{array}$ \\
\hline Keywords: & X-ray topography, ice, dislocation interactions \\
\hline Keywords (user supplied): & scale invariance, internal stresses \\
\hline
\end{tabular}

\section{S) ScholaroNE \\ Manuscript Central}


Ice single crystals were deformed under torsion and dislocation arrangements were analysed by synchrotron topography at ESRF (European Synchrotron Radiation Facility). Profile analysis of topographs reveals a scale invariant character of dislocation arrangement with long-range correlations. Dislocation density gradients are shown to be slightly anti-correlated as the intensity profile is similar to an anti persistent random walk-like signal. This analysis reveals the influence of internal stresses on dislocation arrangement up to the sample scale. Similar observations on reversed torsion experiment, together with strong hardening behaviour, allow to suggest the mechanism of cross-slip of basal dislocations on prismatic planes for interpretation of local dislocation interaction behaviour.

Keywords : dislocation interactions, X-ray topography, ice, scale invariance, internal stresses

\section{Introduction}

The viscoplastic deformation of crystalline materials involves the motion of large numbers of interacting dislocations. Plastic deformation has been shown to be heterogeneous : in most cases the dislocations tend to move cooperatively in groups on a few relatively closely spaced slip-plane clusters rather than individually and independently from each other [1]. This is well known from the observation of the so-called "slip lines" or "slip bands" on the surface of deformed crystals, where slip steps on the surface characterize the collective motion of large numbers of dislocations [2].

In materials with high dislocation densities, dislocation motion is highly intermittent in space and time. At a fixed location, the plastic strain rate is close to zero most of the time, and plastic deformation takes place as isolated "bursts" where the instantaneous strain rate may exceed the average (imposed) strain rate by several orders of magnitude. The motion of dislocations within the active slip volume proceeds in a collective and self organized manner that can not be described in terms of local and uncorrelated relaxations of individual dislocation segments toward configurations of lower energy [1]. Collective movements of dislocations are not restricted to the case of macroscopic plastic instabilities, such as Portevin Le Chatelier type instabilities, but 
constitute a generic feature of the microscopic and mesoscopic dynamics of dislocation systems in materials with high dislocation mobility [3].

Observation of slip steps at the surface [1,2] has often been used to learn about this cooperative motion of dislocations. One can wonder if this information collected at the surface is representative of the physics of deformation within the bulk. X-ray diffraction appears as a powerful technique for such observations as it allows bulk characterization of dislocation arrangements for X-ray transparent materials.

Like in various metals and rocks, deformation in ice crystals has been observed to exhibit large spatio-temporal fluctuations. Nakaya [4] provided macroscopic observations of slip bands on bended single crystals. Mansuy et al. [5] also evidenced the localization of slip in a single crystal deformed in a polycrystalline matrix. Both are macroscopic surface and bulk observations through polarized light, after and during deformation. Acoustic emission analysis performed on ice single crystals [6-8] revealed the scale-free intermittent motion of dislocations through dislocation avalanches. The size distribution of these "slip events", as monitored by acoustic emission, follows a power law [6,7] that characterizes scale invariant systems.

The present work is based on X-ray diffraction analysis of ice single crystals deformed under torsion. The main technique presented here is synchrotron X-ray diffraction topography which allows to detail the dislocation arrangements after deformation and leads to the observation of deformation localization in what could be first called "slip bands". However, a scaling analysis of the observed deformation patterns reveals scale invariant dislocation arrangements for which the identification of "slip bands" is meaningless.

\section{Experimental devices}

Torsion experiments were performed on single crystals artificially grown in the laboratory. Sample characteristics are given in table 1. The initial dislocation density of the sample was estimated to be less than $10^{8} \mathrm{~m}^{-2}$ [9]. The orientation of the samples was chosen to align the torsion axis as close as possible to the c-axis $\left( \pm 1^{\circ}\right)$. Basal planes were therefore parallel to the permanent shear plane. The temperature was maintained at $-15 \pm 1^{\circ} \mathrm{C}$. Applied shear stress on the outer surface of the cylinder was $0.05 \mathrm{MPa}$. A constant torsion torque was applied to each sample. Shear strains reached $0.71 \%$ for sample 1 and $2.69 \%$ for sample 2 . Sample 3 was deformed up to $4.35 \%$ and the torsion was reversed up to $4.25 \%$, to obtain a total shear close to zero. A typical creep curve is given in figure 1 for sample 1 which shows the increase in strain rate during the creep test. The reverse torsion applied on sample 3 was characterized by a strong decrease of the strain rate (figure $2)$.

Squared specimens of $2 \mathrm{~mm}$ in thickness and about $10 \mathrm{~mm}$ in side were collected along the 
diameters of samples 1, 2 and 3. They were studied via synchrotron X-ray diffraction topography at ESRF. Observations were performed on the ID19 beamline, which main features derive from the requirements of having a spectrally and spatially homogeneous, highly coherent beam at the sample position, with (maximum) dimensions $45 \times 15 \mathrm{~mm}^{2}$. X-ray topography is an imaging technique based on Bragg diffraction that provides a two-dimensional intensity mapping of the beam(s) diffracted by a crystal. It records the long-range distortion fields associated with a macroscopic crystal deformation. These distortion fields affect the diffracted intensity, so give "contrast" (nonhomogeneous intensity distribution) in the image. In this way topography is a study of the fine structure of a Bragg spot which contains the information about the departures from the perfect crystal structure, that is the defect structure. High energy (50-120 keV) white beam was used to allow the investigation of bulk ice samples in transmission. Images are recorded on films and scanned to be treated. The film plate resolution ranges from about 1 to several $\mu \mathrm{m}$, but scanning induces a final resolution of about $10 \mu \mathrm{m}$. Vertical slits of $0.1 \mathrm{~mm}$ were positioned between the beam and the sample in order to minimize surface effects on topographs. Topograph on figure $3 \mathrm{a}$ is the diffraction pattern on the prismatic plane for a specimen taken from sample 1 . The long dimension is the height of the specimen, while the width corresponds to the size of the diffracted beam through the $0.1 \mathrm{~mm}$ slit.

We will also briefly present analyses of ice crystals using a hard X-ray diffraction technique as in [10]. For comparison purposes, samples deformed at higher strain under torsion creep (up to $7 \%$ ) and non deformed samples were analysed. These experiments were carried out at the Institute Laue Langevin (ILL) in Grenoble, using an industrial high-voltage X-ray tube $(420 \mathrm{kV})$ and the original Laue hard X-ray technique that allows in situ observation of bulk samples more than $1 \mathrm{~cm}$ thick [11]. The energy range for the white divergent beam is between 100 and $400 \mathrm{keV}$, resulting in wave lengths between 0.03 and $0.12 \AA$. The width of the diffraction peaks is directly related to the lattice distortion [11]. The observed diffraction lines can be analysed with regard to the lattice distortion and the nature of dislocations involved.

[Insert table 1, figure 1, figure 2 and figure 3 around here]

\section{Statistical analyses of the heterogeneity of dislocation arrangements.}

During synchrotron X-ray topography, the diffracted intensity records the long-range distortion field, in our case related to orientation contrast. For our ice samples, it is related to the dislocation density at the origin of the lattice distortion. By nature, the dislocation arrangements revealed by this technique results from "geometrically necessary dislocations", GNDs, only, whereas possible "statistically stored dislocations", SSDs, at the scale below the resolution scale (10 $\mu \mathrm{m})$ are inaccessible (see below further discussion about GNDs and SSDs). Although the relationship 
between diffracted intensity and dislocation density is not straighforward, darker zones of the synchrotron topographs correspond to the higher dislocation density regions. Diffracted intensity variations provide a map of dislocation density variations along the sample, see figure 3a. Sample's c-axis being along the torsion axis, dislocations are expected to be mainly of screw character and to glide on basal planes to accommodate the torsion deformation up to the sample scale [10]. We then only study the diffraction pattern along the [100] prismatic planes with which no extinction were expected for any of the 3 glide systems with a/3<11-20> Burgers vectors. X-ray analyses of the three samples provide global images of the dislocation distribution all over the sample height which was about $10 \mathrm{~mm}$ (see table 1). Dislocation densities appear strongly heterogeneous over the samples, through areas of highly localized distortion, alternating with low distorted areas. Spectral analyses were performed along 1D intensity profiles of the topograph, perpendicular to the basal plane, figure $3 \mathrm{~b}$. The scaling properties of the profiles are found to be independent on the position of the profile along the basal plane. The diffracted intensity is spatially heterogeneous in the sense that it is not distributed around a mean value following a Gaussian distribution, instead it is characterized by a wide distribution and, as shown below, by spatial correlations.

Figure 4 shows the power spectrum of the intensity record for sample 1 (calculated from the fast Fourier transform of the signal). The power spectra have similar behaviour for the three samples. They are characterized by a power law regime over the entire available scale range (bounded by the resolution of the analysis, and by the sample size), $E(f) \sim f^{-\mu}$. These scale ranges extend over more than 2 orders of magnitude, from $15 \mu \mathrm{m}$ to about $7 \mathrm{~mm}$. The power spectrum has an exponent $\mu=1.3 \pm 0.1$ for the three samples. These power laws reveal the scale invariance of the intensity records, that is of the dislocation density arrangement along a line perpendicular to the basal planes. This can also be interpreted as a self-affine character of the dislocation density profiles, with a Hurst's exponent $\mathrm{H}=(\mu-1) / 2 \approx 0.15$. Self-affine properties can be analysed by means of other methods, such as the "variable bandwidth method", but the power spectrum appears as the most reliable method for such low exponents $H$ [12]. This scale invariance has a first important consequence: although dislocation density is strongly heterogeneous, to define "slip bands" is misleading in the present case, as it is scale dependent. Indeed, if we define a slip band as a local maximum on the intensity record, the number of slip bands per unit length, averaged over the entire record, will increase as we increase the resolution of the analysis: more local maxima appear with increasing resolution. This is exactly what can be observed from surface morphology analyses of deformed $\mathrm{Cu}$ polycrystals [2] and $\mathrm{Cd}$ single crystal [13]. Although sample 3 is macroscopically "non deformed", the dislocation distribution keeps the same characteristics as the one of deformed samples. At the microscale, dislocations coming from both torsion directions must interact but the statistical properties of the dislocation arrangement remain the same. [insert figure 4 around here]

Such scale invariant spectra also reveal that the dislocation density is spatially correlated 
over very large distances, with a correlation length of the order of the system (sample) size. This is confirmed by the autocorrelation function of the records shown on figure 5. These autocorrelations slowly decrease with increasing distance $\Delta x$, to fall around nil values for distances of few millimetres. These large scale correlations are the fingerprint of strong interactions between dislocations. Dislocations are neither distributed randomly (as they would for a non-interacting case), nor concentrated on well-defined "slip bands" more or less regularly spaced. Instead, they self-organize into a scale free pattern where the probability to find a large density of dislocations at some place increases in the vicinity of other large density places, with this correlation slowly decreasing with increasing separation. This static, "post-mortem" scale-free pattern is in qualitative agreement with the scale-free pattern of dislocation avalanches recorded by acoustic emission during the creep of ice single crystals [8]. These results clearly evidence that the scale invariance, and therefore the long range correlations, are associated to a deformation induced organization. We do not have ESRF synchrotron topograph of a non deformed sample, but a comparison could be done thanks to hard X-ray analyses performed at ILL on deformed and non deformed samples. The crystals analysed at ILL were deformed under torsion to higher strains (up to 7\%) [10]. Although the spatial definition provided by the technique did not allow to perform a spectral analysis of the diffracted intensity, the autocorrelation functions can be calculated. Figure 6 represents autocorrelation function of diffracted intensity as a function of distance along the sample for a nondeformed and two deformed samples. Long range correlations, as the one observed for the ESRF samples, clearly appear on the deformed samples, while the intensity record of the non deformed sample is essentially uncorrelated from very short distance. Spatial correlation and therefore scale invariance, are then induced by plastic deformation process. [insert figure 5 and figure 6 around here]

To go further, an analogy with random walk type of signal (random walks were first developed in the context of Brownian motion, see e.g. [14]), is worth pursuing. A one-dimensional random walk $y(x)$ is easily constructed, started from an initial value $y_{0}=y\left(x_{0}\right)$, by successive additions of increments $\Delta y: y(x+\Delta x)=y_{0}+\Delta y_{1}, y(x+2 \Delta x)=y(x+\Delta x)+\Delta y_{2}$, and so on... For "classical" random walk, the increments $\Delta y_{i}$ are chosen independently from a Gaussian distribution with zero mean. The random walk itself is, by construction, correlated over (theoretically) infinite distances $x$. In case of diffusion and Brownian motion, the axis $x$ refers to a time and the axis $y$ to a distance. In our case, $x$ is a distance along a line perpendicular to the basal planes and $y$ is our intensity $I$, i.e. a proxy of dislocation density. On figure 7 are shown the distributions of the increments $\Delta I$ for sample 1. In our case, the increments represent dislocation density gradients. The distribution shows, compared to Gaussian statistics, a slight deficit of intermediate (absolute) values and a excess of both small and large values. In case of a time record, this reveals an intermittent character of the signal with some anomalously abrupt changes in between anomalously quiescent periods, as 
it is observed in the so-called anomalous diffusion [15]. In our case this would mean an "anomalously" strong heterogeneity of dislocation density, with narrow zones characterized by large dislocation density gradients, separated by more homogeneous regions. Note however that this departure from normal behaviour is significant but small. Another strong characteristic of classical random walk is the independence of the increments, with a correlation length equal to 0 . In this case, the power spectrum of the random walk should scale as $f^{-2}$ [14]. The exponents observed for our samples $(\mu \cong 1.3$ ) are lower than 2 . Our signals could then be analysed as the so-called antipersistent fractional Brownian motion introduced by Mandelbrot and Van Ness [16]. Anti-persistent records are characterized by a signal (the dislocation density in our case) where an increase between $x$ and $x+\Delta x$ (positive increment) is expected, in the probabilistic sense, to be followed by a decrease (negative increment) for the next value of $x$. Our dislocation density records are then anti-persistent, meaning that the dislocation density gradients are slightly anti-correlated, as our sample behaviour show a large departure from classical random walk, with an exponent $\mu$ significantly smaller than 2 . This anti-correlation is illustrated on figure 8 where (i) the gradient autocorrelation function is negative for very small $\Delta x$ and (ii) the autocorrelation of the absolute value of the gradient signal remains above zero for $\Delta x$ as large as $1 \mathrm{~mm}$. To summarize, this detailed analysis reveals strong heterogeneity of dislocation density distribution, where large dislocation density gradients are clustered within more homogeneous zones. Furthermore, it appears that dislocation density gradients are slightly anti-correlated, meaning that a positive gradient is more likely surrounded by a negative gradient. These last results would reveal a more local influence of internal stresses at the scale down to dislocation density gradient localization. [insert figure 7 and figure 8 around here]

\section{Discussion}

The presented statistical analysis of X-ray diffraction patterns from ice single crystals deformed under torsion details dislocation arrangements along a line perpendicular to the basal planes. This statistical analysis reveals scale invariant, anti-persistent patterns with long-range correlations. The scale-free pattern is the fingerprint of a self-organization of dislocations during deformation, which interact through their elastic stress fields up to the sample scale. The anti-persistent character of the diffracted intensity signal reveals the nature of correlations for dislocation density gradients which appear to be slightly anti-correlated at a local scale. However, the very nature of this complex dynamics is not easy to understand and/or to model. In particular, our analyses revealed the 1D profiles of patterns that are the results of a 3D complex self-organization.

The analysed diffraction patterns reveal the lattice distortion and then, the GNDs associated with this distortion. Considering torsion deformation conditions of the present experiments, the easy slip system (basal glide) of the main dislocations which are of screw character, is activated. 
We then expect that all the dislocations mainly accommodate the imposed strain, and then are GNDs whatever the considered scale [10]. Consequently, dislocation density, in this deformation configuration, is a direct proxy of the torsion strain.

The distinction between GNDs and SSDs is a matter of scale of observation. Dislocations can be considered as SSDs if they do not, in average and at a given scale, contribute to lattice distortion, that is, if their effect can be homogenised (at the crystal lattice scale, any dislocation is a GND). The scale invariance of the lattice distortion signal reveals that dislocation patterns and their effects on the lattice distortion and strain can not be homogenised in the scale range considered (10 $\mu \mathrm{m}-10 \mathrm{~mm})$. For scales below the resolution scale $(10 \mu \mathrm{m})$, nothing can be deduced from our measurements, but observations of slip steps at the surface of $\mathrm{Cu}$ polycrystals [2] reveal scale invariant patterns over lower scale range $(10 \mathrm{~nm}-2 \mathrm{~mm})$. In other words, the dislocation density becomes scale dependant, and the notion of SSDs can be questioned, at least for the deformation and materials considered.

Ice is characterized by a relatively low Peierls stress. A low lattice friction, i.e. a relatively high dislocation mobility, is a condition for long range interactions between dislocations to occur. The acoustic emission data, induced by dislocation motion during ductile deformation of ice single crystals evidenced the collective dislocation dynamics as being a critical phenomenon [7]. The long-range correlation and scale invariant behaviour observed in the torsion deformed single crystals are in agreement with this framework.

Assuming that dislocation sources are infinite at the surface of the single crystal, dislocation distribution has to be explained taking into account long range internal stresses. In torsion, the long range internal stress at the origin of the scale invariance must be related to the interaction between screw dislocations of the same sign. Cross slip of screw basal dislocations on prismatic planes can then be invoked to explain the dislocation arrangement. Shear stress in prismatic planes necessary for cross slip to occur is provided by the internal stress field induced by basal screw dislocations. The hardening occurring when reversed torsion is applied to a deformed sample (case of sample 3) would be induced by interactions between screw dislocations of opposite signs. These interactions can only occur if cross slip induces jog formation which prevent dislocations to glide back when reversed torsion is applied. Cross slip induces irreversibility of dislocation motion, explaining that the scale invariant pattern is maintained during reverse torsion creep, although macroscopic strain is nil.

\section{Conclusions}

Dislocation arrangements have been characterized in ice single crystals deformed in torsion (well oriented for basal slip) thanks to synchrotron X-ray topography. The dislocation arrangements 
reveal scale invariant, anti-persistent patterns with long-range correlations. Long-range correlations reveal the influence of internal stresses up to the sample scale, and evidence of anti-persistence provide information about the anti-correlated nature of local dislocation density gradient interactions. Over the scale range considered, and much below for other materials [2], dislocation patterns, and their effects on lattice distortion and strain, can not be homogenised. The notion of SSDs can then be questionned, at least considered as scale dependant, in this context. Furthermore, internal stresses appear of major impact when considering dislocation dynamics in ice. Indeed, the reverse torsion experiment points out a strong hardening that can be explained by interaction between screw dislocations of opposite signs. Cross-slip of dislocations on non-basal plane would induce jog formation and prevent motion of dislocations. Although shear at the macroscopic scale can be suppressed by reverse torsion, at the microscopic scale dislocation arrangements keep the statistical properties of deformed samples, i.e. shear annihilation only occurs at the macroscopic, sample, scale and not below. 


\section{Acknowledgements \\ We are very thankful to ESRF organisation, and more particularly to Jürgen Härtwig and José Baruchel from ID19 beamline. We would also like to thank Juliette Chevy and Marc Fivel for fruitful discussions. And many thanks to the two referees for valuable comments which helped improving the quality of the paper.}


[1] H. Neuhauser, in Dislocations in Solids, edited by F.R.N. Nabarro (North Holland, Amsterdam 1983) pp. 319.

[2] M. Zaiser, F. Madani, V. Koutsos and E.C. Aifantis, Phys. Rev. Let., 93 pp. 1-4 (2004).

[3] M. Zaiser and A. Seeger in Dislocations in Solids, edited by F.R.N. Nabarro and M.S. (Duesbury, Elsevier 2002) pp. 1-100.

[4] U. Nakaya, in Mechanical properties of single crystals of ice (US Army Snow Ice and Permafrost Res. Establish 1958) Report 28.

[5] P.Mansuy, A. Philip and J. Meyssonnier, J. Phys. IV France 11, pp. 267-274 (2001).

[6] J. Weiss and J.R. Grasso, J. Phys. Chem. 101 pp. 6113-6117 (1997).

[7] M. C. Miguel, A. Vespignani, S. Zapperi, J. Weiss and J.R. Grasso, Nature 410 pp. 667-671 (2001).

[8] J. Weiss and D. Marsan, Science 299(5603) pp. 89-92 (2003).

[9] M. Montagnat, P. Duval, P. Bastie, B. Hamelin, O. Brissaud, M. de Angelis, J.R. Petit, V.Ya. Lipenkov, C. R. Acad., Paris 333 pp. 419-425 (2001).

[10] M. Montagnat, P. Duval, P. Bastie, B. Hamelin, Scripta Mater. 49 pp. 411-415 (2003).

[11] B. Hamelin and P. Bastie, J. Phys. IV France 8 pp. 3-8 (1998).

[12] J. Schmittbuhl, J.P. Vilotte and S. Roux, Phys. Rev. E 51 (1) pp131-147 (1995).

[13] B. Sprusil and F. Hnilica, Czech. J. Phys. 35 pp. 897-900 (1985).

[14] J. Feder, Fractals, edited by Plenum Press, New York (1988).

[15] J. P. Bouchaud and A. Georges, Physics Reports 195 pp. 127-129 (1990).

[16] B. Mandelbrot and J. Van Ness, SIAM Review 10(4) pp. $422-437$ (1968). 


\section{Figure captions :}

Figure 1 : Torsion creep curve, sample 1 . Total deformation reached is $0.71 \%$ at the cylinder surface.

Figure 2 : Direct and reversed torsion creep curves, sample 3. Direct torsion reached 4.35\%, reversed torsion reached $4.25 \%$. Applied stress is represented.

Figure 3 : a) Synchrotron X-ray topograph obtained on a slice of sample 1 (c-axis, and then torsion direction is represented). b) Intensity profile (arbitrary units) obtained along this topograph.

Figure 4: Power spectrum of the intensity record along a profile taken on the synchrotron X-ray topograph of sample 1 . The 1.3 slope is represented. The white dots are averages over bins regularly spaced in logarithmic scale.

Figure 5 : Autocorrelation functions of the intensity spectra along profiles taken on the synchrotron X-ray topographs of sample 1 (thick line) and sample 2 (thin line).

Figure 6: Autocorrelation functions of hard X-ray intensity records from 3 samples. Dashed line: deformed, dots: non-deformed, and solid line: deformed and reversed.

Figure 7 : Distribution of intensity increments $\Delta \mathrm{I}$ for sample 1 (thick line). For comparison, a Gaussian distribution with the same statistic characteristics is shown.

Figure 8 : Autocorrelation functions of the strain gradients signals for sample 1. Autocorrelation is drawn for both absolute value of the signal (thick line) and the total signal (thin line). 


\begin{tabular}{|c|c|c|c|c|}
\hline Sample & Diameter $(\mathbf{m m})$ & Height $(\mathbf{m m})$ & Shear stress (MPa) & $\begin{array}{c}\text { Maximum shear } \\
\text { strain (\%) }\end{array}$ \\
\hline 1 & 29 & 47 & 0.05 & 0.71 \\
\hline 2 & 28 & 42 & 0.05 & 2.69 \\
\hline 3 & 49 & 60 & 0.05 & $+4.35-4.25$ \\
\hline
\end{tabular}

Table 1 : Characteristics of samples deformed under torsion 
Figure 1 : Torsion creep curve, sample 1 . Total deformation reached is $0.71 \%$ at the cylinder surface. $260 \times 203 \mathrm{~mm}(300 \times 300 \mathrm{DPI})$ 


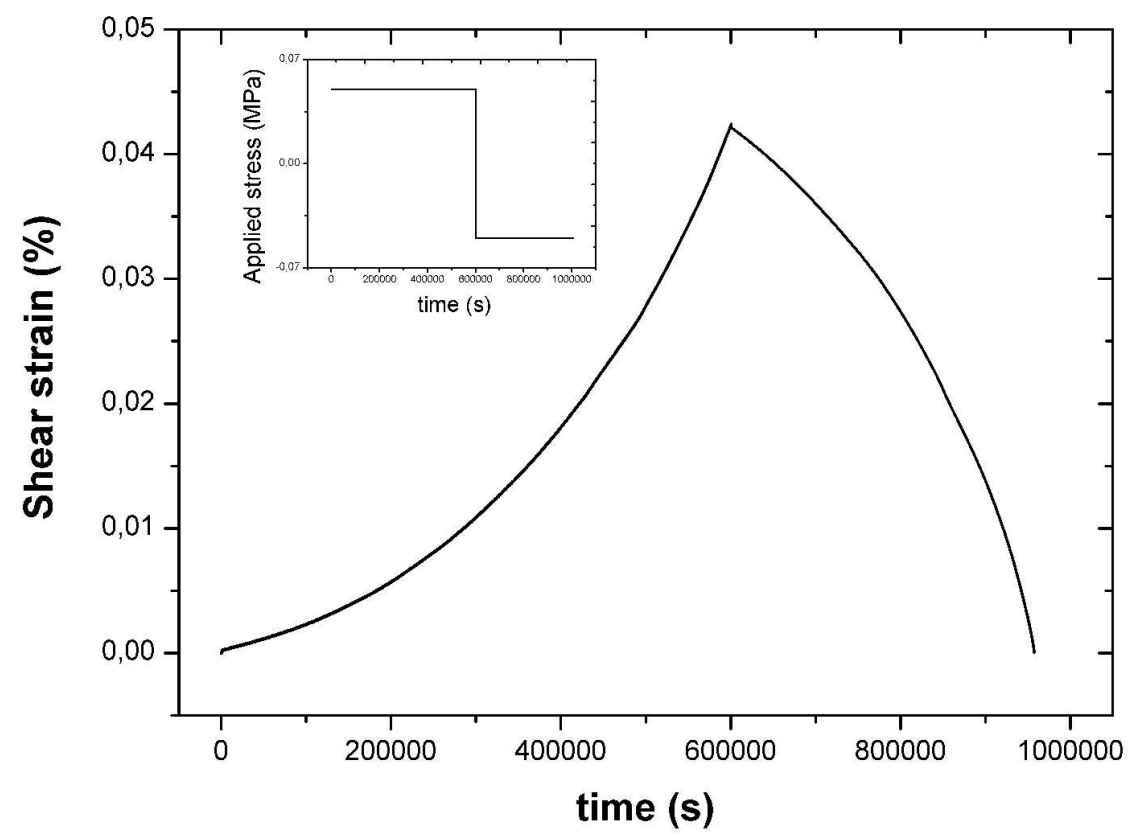

Figure 2 : Direct and reversed torsion creep curves, sample 3. Direct torsion reached $4.35 \%$, reversed torsion reached $4.25 \%$. Applied stress is represented. $273 \times 202 \mathrm{~mm}(300 \times 300 \mathrm{DPI})$ 

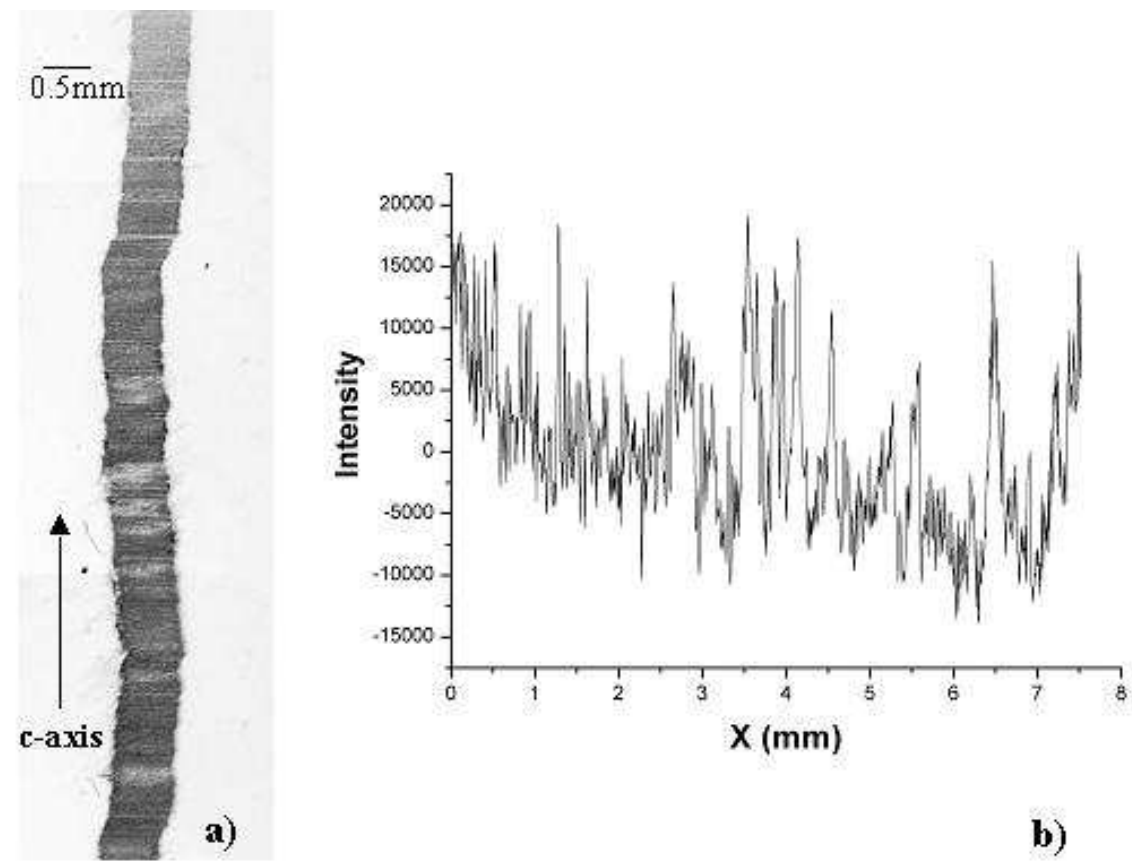

a)

b)

Figure $3:$ a) Synchrotron X-ray topograph obtained on a slice of sample 1 (c-axis, and then torsion direction is represented). b) Intensity profile (arbitrary units) obtained along this topograph. $48 \times 36 \mathrm{~mm}(300 \times 300 \mathrm{DPI})$ 
Figure 4: Power spectrum of the intensity record along a profile taken on the synchrotron X-ray topograph of sample 1 . The 1.3 slope is represented. The white dots are averages over bins regularly spaced in logarithmic scale. $279 \times 215 \mathrm{~mm}(150 \times 150 \mathrm{DPI})$ 
Figure 5 : Autocorrelation functions of the intensity spectra along profiles taken on the synchrotron X-ray topographs of sample 1 (thick line) and sample 2 (thin line). $279 \times 215 \mathrm{~mm}(150 \times 150 \mathrm{DPI})$ 


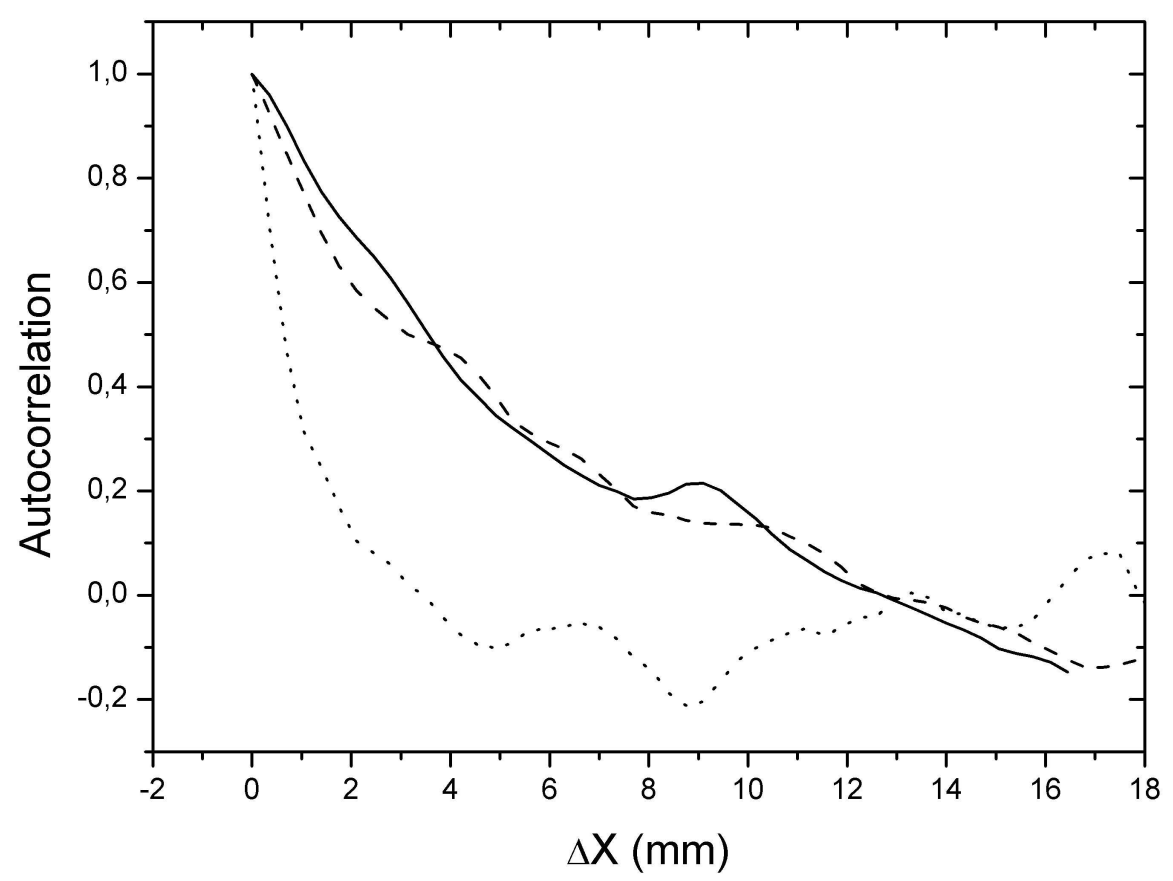

Figure 6 : Autocorrelation functions of hard X-ray intensity records from 3 samples. Dashed line: deformed, dots: non-deformed, and solid line: deformed and reversed. $258 \times 199 \mathrm{~mm}(300 \times 300 \mathrm{DPI})$ 
Figure 7 : Distribution of intensity increments $\Delta \mathrm{I}$ for sample 1 (thick line). For comparison, a Gaussian distribution with the same statistic characteristics is shown. $279 \times 215 \mathrm{~mm}(150 \times 150 \mathrm{DPI})$ 


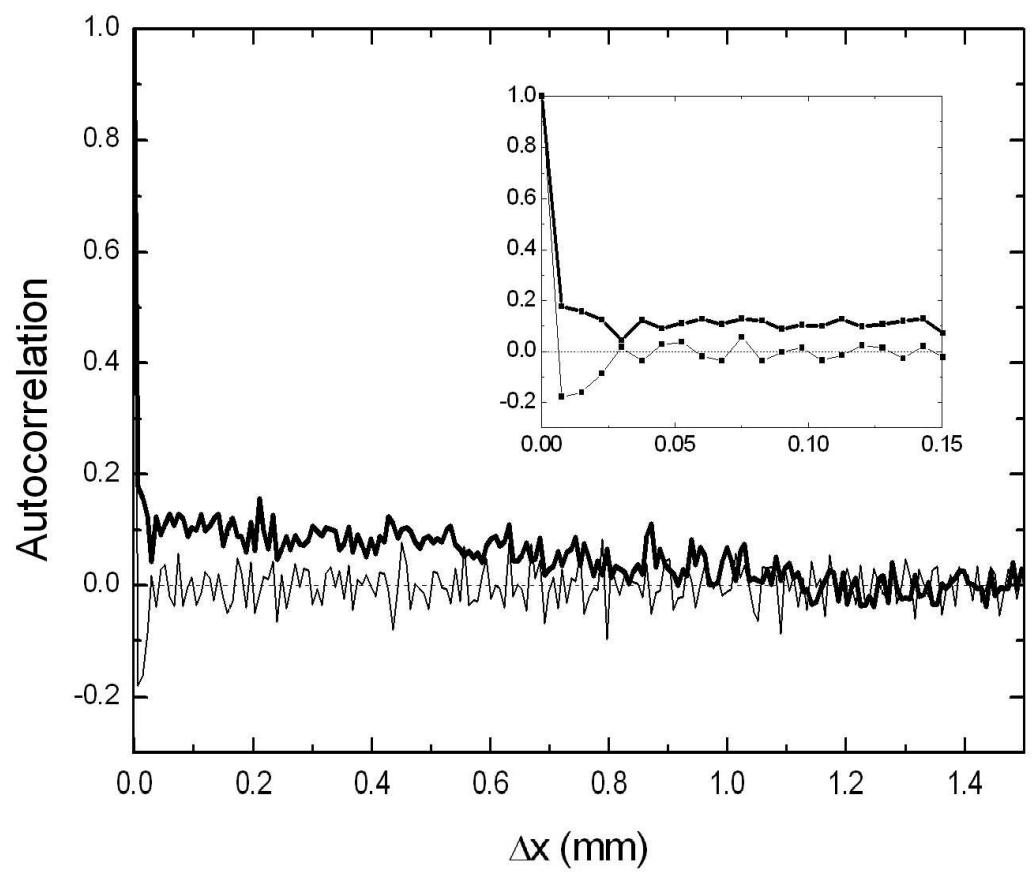

Figure 8 : Autocorrelation functions of the strain gradients signals for sample 1 . Autocorrelation is drawn for both absolute value of the signal (thick line) and the total signal (thin line). $279 \times 215 \mathrm{~mm}(150 \times 150 \mathrm{DPI})$ 